\title{
One-pot synthesis of 2,4,5-tri-substituted-1H-imidazoles promoted by trichloromelamine
}

\author{
BiBi Fatemeh Mirjalili ${ }^{a^{*}}$, Abdolhamid Bamoniri ${ }^{\mathrm{b}}$ and Najmeh Mohaghegh ${ }^{\mathrm{a}}$
}

${ }^{a}$ Department of Chemistry, College of Science, Yazd University, Yazd, P.O.Box 8915813149,, Iran ${ }^{b}$ Department of Organic Chemistry, Faculty of Chemistry, University of Kashan, Kashan, I. R. Iran

\section{H R O N I C L E}

Article history:

Received June 25, 2012

Received in Revised form

November 6, 2012

Accepted 8 December 2012

Available online

10 December 2012

\section{Keywords:}

2,4,5-Trisubstituted Imidazoles

1,2-Diketones

Trichloromelamine

Aldehydes

Positive chlorine \begin{abstract}
A B S T R A C T
2,4,5-Trisubstituted imidazoles have many pharmaceutical properties and can be prepared via reaction of 1,2-diketones with aldehydes in the presence of an acidic catalyst. In this work, we have prepared 2,4,5-trisubstituted imidazoles in the presence of trichloromelamine as a source of positive chlorine. Short reaction times, high yield, simplicity of operation and easy work-up are some advantages of this method.
\end{abstract}

\section{Introduction}

2,4,5-Trisubstituted imidazoles are used as fungicides, herbicides, plant growth regulators, inhibitors of IL-1 or p38 MAP kinase, CB1 cannabinoid receptor antagonists ${ }^{1}$, antibacterial ${ }^{2}$, antitumor $^{3}$, and glucagon receptors ${ }^{4}$.

There are several reported methods for the synthesis of 2,4,5-trisubstitutedimidazoles such as the hetero-Cope rearrangement ${ }^{5}$, and four-component condensation of arylglyoxals, primary amines, carboxylic acids and isocyanides on Wang resin 6 . Recently, the synthesis of 2,4,5-trisubstituted imidazoles has been catalyzed by $\mathrm{ZrCl}_{4}{ }^{7}$, ionic liquid ${ }^{8}$, silica gel or Zeolite $\mathrm{HY}^{9}, \mathrm{InCl}_{3} \cdot 3 \mathrm{H}_{2} \mathrm{O}^{10}$, $\mathrm{DABCO}^{11}$, PEG-400 ${ }^{12}$, Wells-Dawson heteropolyacid supported on silica $\left(\mathrm{WD} / \mathrm{SiO}_{2}\right)^{13}$,

* Corresponding author. Fax: +983518210644

E-mail addresses: fmirjalili@yazduni.ac.ir (B.F. Mirjalili)

(C) 2013 Growing Science Ltd. All rights reserved. doi: $10.5267 /$ j.ccl.2012.12.001 
[EMIM] OAc ${ }^{14}, \mathrm{NaH}_{2} \mathrm{PO}_{4}{ }^{15}, \mathrm{~N}$-methyl-2-pyrrolidone hydrogen sulfate ${ }^{16}, \mathrm{~K} 10^{17}, \mathrm{ZMS}-5^{17}$, sulfated zirconia ${ }^{17}, n-\mathrm{Bu}_{4} \mathrm{NBr}^{18},\left(\mathrm{NH}_{4}\right)_{6} \mathrm{Mo}_{7} \mathrm{O}_{24} \cdot 4 \mathrm{H}_{2} \mathrm{O}^{19}$, nano $\mathrm{MgO}^{20}$ and $\mathrm{KH}_{2} \mathrm{PO}_{4}{ }^{21}$.

$N$-halo reagents with high active $N$-X bond have the potential to promote some important reactions. Trichloromelamine (TCM) as a common $N$-halo reagent is used as bleaching agents, disinfectants and bactericides and promoted some organic reactions such as $N$-nitrosation of secondary amines $^{22}$, oxidation of alcohols ${ }^{23,24}$, and trimethylsilylation of hydroxyl group ${ }^{25}$.

\section{Results and disscusion}

In continuation of our investigations on solid acids in organic synthesis ${ }^{26-30}$, we report here on applying trichloromelamine as an efficient $\mathrm{Cl}^{+}$catalyst source for synthesis of 2,4,5-trisubstituted imidazoles via reaction of benzil, aldehydes and ammonium acetate. For investigation the efficiency of trichloromelamine in preparation of 2,4,5-trisubstituted imidazoles, we have examined the reaction of benzaldehyde $(1 \mathrm{mmol})$, benzil $(1 \mathrm{mmol})$ and ammonium acetate $(2 \mathrm{mmol})$ as model reaction. The reaction in different conditions in the presence of trichloromelamine revealed that the best condition was $0.07 \mathrm{~g}$ of trichloromelamine under solvent-free condition at $110^{\circ} \mathrm{C}$ (Entry 2, Table 1).

Table 1 Synthesis of 2,4,5-triphenyl-1H-imidazole under various conditions ${ }^{\mathrm{a}}$

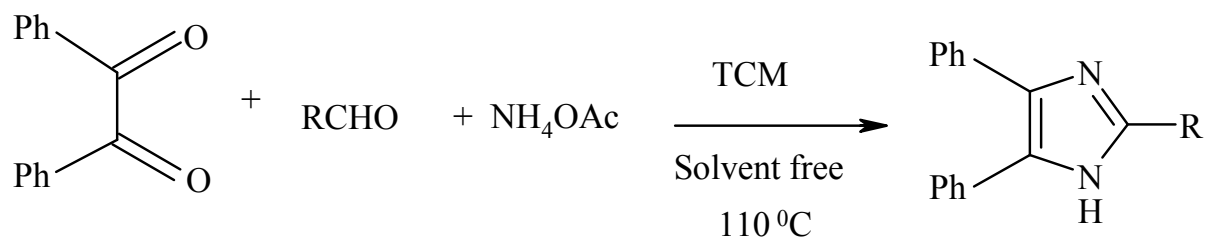

\begin{tabular}{|c|c|c|c|c|c|}
\hline Entry & Reagent (g) & Condition & Time $(\mathrm{h})$ & Yield $(\%)^{b}$ & Ref. \\
\hline 1 & $\mathrm{TCM}(0.05)$ & solvent free/ $110^{\circ} \mathrm{C}$ & 1 & 87 & - \\
\hline 2 & TCM (0.07) & solvent free/ $110^{\circ} \mathrm{C}$ & 1 & 92 & - \\
\hline 3 & $\operatorname{TCM}(0.1)$ & solvent free/ $110^{\circ} \mathrm{C}$ & 1 & 92 & - \\
\hline 4 & $\operatorname{TCM}(0.1)$ & $n$-hexan/ reflux & 24 & - & \\
\hline 5 & $\mathrm{P}_{2} \mathrm{O}_{5} / \mathrm{SiO}_{2}(0.1)$ & solvent free/ $110^{\circ} \mathrm{C}$ & 3 & 90 & 31 \\
\hline 6 & [EMIM]OAc (0.017) & ultrasound/ r.t. & 0.7 & 87 & 14 \\
\hline 7 & $\mathrm{NaH}_{2} \mathrm{PO}_{4}(0.33 \mathrm{mmol})$ & solvent free/ $120^{\circ} \mathrm{C}$ & 0.5 & 99 & 15 \\
\hline 8 & $\mathrm{DABCO}(0.7 \mathrm{~mol} \%)$ & $t-\mathrm{BuOH} / 65^{\circ} \mathrm{C}$ & 12 & 92 & 11 \\
\hline 9 & $\begin{array}{l}N \text {-methyl 2-pyrrolidone hydrogen } \\
\text { sulfate }(0.08)\end{array}$ & solvent free/ $100^{\circ} \mathrm{C}$ & 1.5 & 87 & 16 \\
\hline 10 & $\mathrm{~K} 10(0.01)$ & EtOH/ Reflux & 1.5 & 85 & 17 \\
\hline 11 & ZMS-5 (0.01) & EtOH/ Reflux & 1.8 & 84 & 17 \\
\hline 12 & Sulfated zirconia, SZ (0.01) & EtOH/ Reflux & 1.6 & 82 & 17 \\
\hline 13 & $n-\mathrm{Bu}_{4} \mathrm{NBr}(0.032)$ & $i-\mathrm{PrOH} / 82^{\circ} \mathrm{C}$ & 0.3 & 95 & 18 \\
\hline 14 & $\left(\mathrm{NH}_{4}\right)_{6} \mathrm{Mo}_{7} \mathrm{O}_{24} \cdot 4 \mathrm{H}_{2} \mathrm{O}(0.12 \mathrm{~g})$ & solvent free/ MW & 0.16 & 94 & 19 \\
\hline 15 & Nano $\mathrm{MgO}(0.008 \mathrm{~g})$ & solvent free/ $100^{\circ} \mathrm{C}$ & 0.5 & 94 & 20 \\
\hline 16 & $\mathrm{KH}_{2} \mathrm{PO}_{4}(0.5 \mathrm{~mol} \%)$ & EtOH/ Reflux & 0.6 & 93 & 21 \\
\hline
\end{tabular}

Benzil, ammonium acetate and various aldehydes were then used as substrates for the synthesis of 2,4,5-tri substituted imidazoles (Table 2, scheme 1). Most of the products were known and identified by spectroscopic data and comparison with literature. 


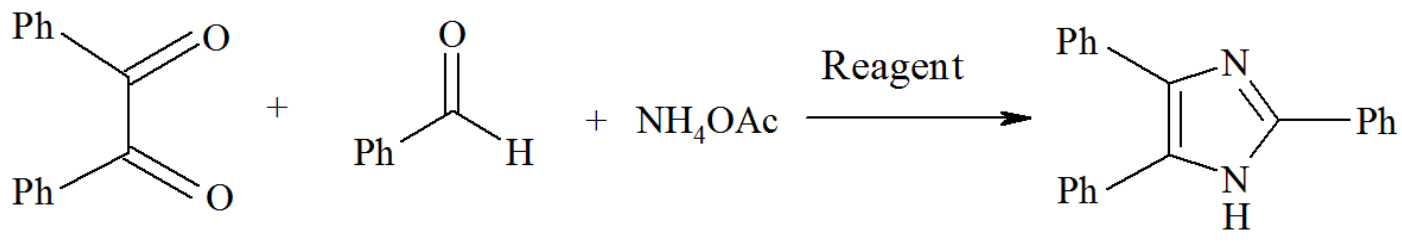

Scheme 1: Synthesis of 2,4,5-tri substituted imidazoles in the presence of trichloromelamine (TCM).

Table 2 Synthesis of 2,4,5-triaryl (alkyl)- $1 \mathrm{H}$-imidazoles promoted by trichloromelamine ${ }^{\mathbf{a}}$

\begin{tabular}{|c|c|c|c|c|c|c|}
\hline \multirow{2}{*}{ Entry } & \multirow{2}{*}{$\mathrm{R}$} & \multirow{2}{*}{ time(h) } & \multirow{2}{*}{ yield $(\%)^{b}$} & \multicolumn{2}{|c|}{ m.p. ${ }^{\circ} \mathrm{C}$} & \multirow{2}{*}{ ref } \\
\hline & & & & obtained & reported & \\
\hline 1 & $\mathrm{C}_{6} \mathrm{H}_{5}$ & 1 & 90 & $274-275$ & $275-276$ & 14 \\
\hline 2 & $4-\left(\mathrm{CH}_{3}\right)_{2} \mathrm{CHC}_{6} \mathrm{H}_{4}$ & 1 & 94 & $253-255$ & - & - \\
\hline 3 & $4-\mathrm{CH}_{3} \mathrm{OC}_{6} \mathrm{H}_{4}$ & 1.5 & 90 & $226-228$ & $227-228$ & 18 \\
\hline 4 & $2-\mathrm{CH}_{3} \mathrm{OC}_{6} \mathrm{H}_{4}$ & 1.5 & 88 & $209-211$ & $207-208$ & 14 \\
\hline 5 & $2-\mathrm{ClC}_{6} \mathrm{H}_{4}$ & 2 & 89 & 197-199 & 197-199 & 14 \\
\hline 6 & 4- $\mathrm{ClC}_{6} \mathrm{H}_{4}$ & 3.5 & 88 & $261-263$ & $260-262$ & 17 \\
\hline 7 & $2,4-\mathrm{Cl}_{2} \mathrm{C}_{6} \mathrm{H}_{3}$ & 11 & 78 & $174-175$ & $174-175$ & 16 \\
\hline 8 & $4-\mathrm{BrC}_{6} \mathrm{H}_{4}$ & 2.5 & 90 & $264-265$ & $263-265$ & 19 \\
\hline 9 & $2-\mathrm{NO}_{2} \mathrm{C}_{6} \mathrm{H}_{4}$ & 6.5 & 87 & $228-230$ & $230-231$ & 31 \\
\hline 10 & $3-\mathrm{NO}_{2} \mathrm{C}_{6} \mathrm{H}_{4}$ & 3 & 86 & $268-269$ & $269-271$ & 19 \\
\hline 11 & $4-\mathrm{NO}_{2} \mathrm{C}_{6} \mathrm{H}_{4}$ & 7 & 89 & $237-238$ & $239-242$ & 14 \\
\hline 12 & $\left(\mathrm{CH}_{2} \mathrm{O}\right)_{3}$ & 3 & 43 & $101-103$ & - & - \\
\hline 13 & $3,4-(\mathrm{HO}){ }_{2} \mathrm{C}_{6} \mathrm{H}_{3}$ & 4.5 & - & $270-271$ & 272 & 18 \\
\hline
\end{tabular}

${ }^{\mathrm{a}}$ The ratio of benzil (mmol): aldehyde $(\mathrm{mmol})$ :ammonium acetate $(\mathrm{mmol})$ : trichloromelamine $(\mathrm{g})$ is 1:1:2:0.07

${ }^{\mathrm{b}}$ Isolated yield

For synthesis of two substituted imidazole, we have used 1,3,5-trioxane as formaldehyde source (Scheme 2).<smiles>O=C(C(=O)c1ccccc1)c1ccccc1</smiles>

Scheme 2: Synthesis of 4, 5-diphenyl imidazole in the presence of trichloromelamine (TCM).

The presence of one broad singlet signal in $\delta=7.22 \mathrm{ppm}$, proves the formation of 4,5-diphenyl imidazole. According to our investigation about the mechanism of 2,4,5-trisubstituted imidazole synthesis in the presence of trichloromelamine, we have found that one or two $\mathrm{NHCl}$ group in this reagent converts to $\mathrm{NH}_{2}$ group. The reusability of trichloromelamine in this reaction is 2 or 3 times. Thus, we have proposed a mechanism for preparation of 2,4,5-trisubstituted imidazole in the presence of trichloromelamine as positive chlorine catalyst in scheme 3 .

\section{Conclusion}

In conclusion, we have demonstrated a simple method for the synthesis of 2,4,5-trisubstituted imidazoles using trichloromelamine as an eco-friendly, inexpensive, and efficient reagent. Short reaction times, high yield, simplicity of operation and easy work-up are some advantages of this method. 

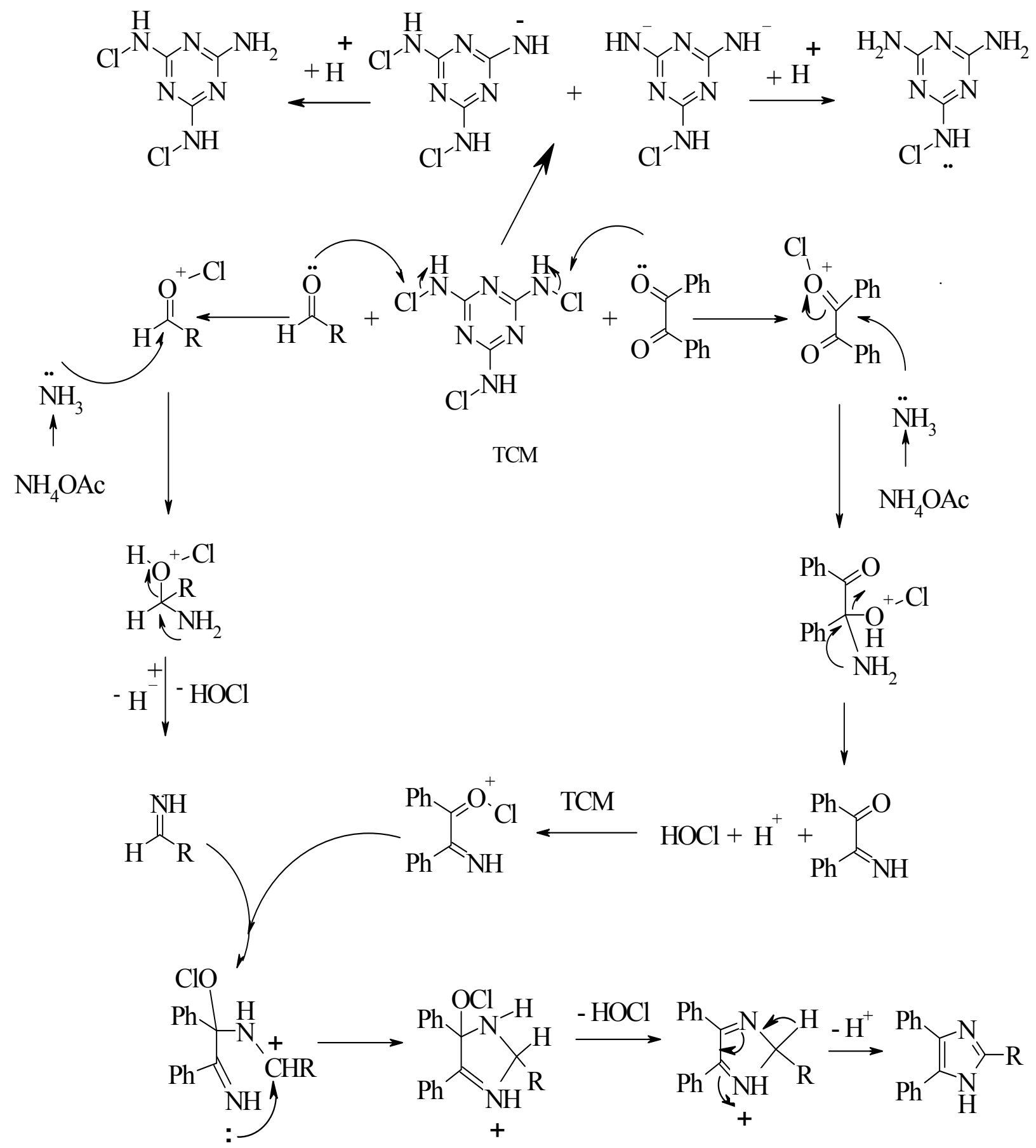

Scheme 3. A proposed mechanism for the synthesis of 2,4,5-trisubstituted imidazoles in the presence of TCM.

\section{Acknowledgment}

The Research Council of Yazd University,Central Iron Ore and Strategic of Research Centre gratefully acknowledged for the financial support for this work. 


\section{Experimental}

The chemicals were used without any additional purification. The products were characterized by FT-IR (ATR), ${ }^{1}$ H-NMR, and a comparison of their physical properties with those reported in the literature. FT-IR (ATR) spectra were run on a Bruker, Eqinox 55 spectrometer. A Bruker (DRX-400 Avance) NMR was used to record the ${ }^{1} \mathrm{H}-\mathrm{NMR}$ spectra. Elemental analyses were done by Costech ECS 4010 CHNS-O analyser.

General procedures for the synthesis of 2,4,5-trisubstituted imidazoles in the presence of trichloromelamine

A mixture of benzil ( $1 \mathrm{mmol})$, aldehyde $(1 \mathrm{mmol})$, ammonium acetate $(2 \mathrm{mmol})$ and trichloromelamine $(0.07 \mathrm{~g})$ was heated with stirring at $110{ }^{\circ} \mathrm{C}$. After completion of the reaction (monitored by TLC), acetone was added to the cold reaction mixture and the catalyst was recovered by filtration and washed with acetone $(2 \times 5 \mathrm{ml})$. By adding water to the filtrate, a milky to yellow solid was obtained. The dried solid was washed with hot $n$-hexane to obtain highly pure product. Most of the products were known and identified by comparison of their physical and spectral data with those of authentic samples.

\section{Selected spectroscopic data}

\section{2,4,5-Triphenyl-1H-imidazole (Table 2, entry 1)}

FT-IR: $\bar{v}(\mathrm{KBr})=3443(\mathrm{~N}-\mathrm{H}), 3038,1602,1504,1461,766,697 \mathrm{~cm}^{-1} .{ }^{1} \mathrm{H}-\mathrm{NMR}(500 \mathrm{MHz}, \mathrm{DMSO}-$ $\left.\mathrm{d}_{6}\right): 12.68(\mathrm{~s}, 1 \mathrm{H}, \mathrm{N}-\mathrm{H}), 8.09\left(\mathrm{~d},{ }^{3} \mathrm{~J}=7.5 \mathrm{~Hz}, 2 \mathrm{H}, \mathrm{Ar}-\mathrm{C}-\mathrm{H}\right), 7.38\left(\mathrm{t},{ }^{3} \mathrm{~J}=7.2 \mathrm{~Hz}, 2 \mathrm{H}, \mathrm{Ar}-\mathrm{C}-\mathrm{H}\right), 7.31$ (brs, 2 H, Ar-C-H), 7.23 (brs, $1 \mathrm{H}$, Ar-C-H), 7.45-7.55 (m, 8H, Ar-C-H) ppm.

\section{2-(4-Isopropyl phenyl)- 4, 5-diphenyl-1H-imidazole (Table 2, entry 2)}

FT-IR: $\bar{v}(\mathrm{KBr})=3029,2961,1602,1490,837,765,696 \mathrm{~cm}^{-1} .{ }^{1} \mathrm{H}-\mathrm{NMR}\left(500 \mathrm{MHz}, \mathrm{DMSO}-\mathrm{d}_{6}\right)$ : 12.60 (s, $1 \mathrm{H}, \mathrm{N}-\mathrm{H}), 8.01$ (d, $\left.{ }^{3} \mathrm{~J}=8.2 \mathrm{~Hz}, 2 \mathrm{H}, \mathrm{Ar}-\mathrm{C}-\mathrm{H}\right), 7.35$ (d, $\left.{ }^{3} \mathrm{~J}=8.2 \mathrm{~Hz}, 2 \mathrm{H}, \mathrm{Ar}-\mathrm{C}-\mathrm{H}\right), 7.38-7.52$ $(\mathrm{m}, 10 \mathrm{H}, \mathrm{Ar}-\mathrm{C}-\mathrm{H}), 2.94$ (sep, $\left.{ }^{3} \mathrm{~J}=6.9 \mathrm{~Hz}, 1 \mathrm{H}, \mathrm{C}-\mathrm{H}\right), 1.25\left(\mathrm{~d},{ }^{3} \mathrm{~J}=6.9 \mathrm{~Hz}, 6 \mathrm{H}, 2 \mathrm{CH}_{3}\right) \mathrm{ppm}$. Elemental analysis. Found, \%: C 85.03; $\mathrm{H} 6.61 ; \mathrm{N} 8.36 . \mathrm{C}_{24} \mathrm{H}_{22} \mathrm{~N}_{2}$. Calculated, \%: C 85.17; H 6.55; N 8.28

\section{2-(2-Chlorophenyl)-4, 5-diphenyl-1H-imidazole (Table 2, entry 5)}

FT-IR: $\bar{v}(\mathrm{KBr})=3026,1600,1482,1069,826,766,695 \mathrm{~cm}^{-1},{ }^{1} \mathrm{H}-\mathrm{NMR}\left(400 \mathrm{MHz}, \mathrm{DMSO}-\mathrm{d}_{6}, \mathrm{ppm}\right)$ : 7.27 (brs, $3 \mathrm{H}$ ), 7.42 (brs, $5 \mathrm{H}$ ), 7.49 (brs, $3 \mathrm{H}$ ), 7.67 (brs, $2 \mathrm{H}$ ), 8.55 (brs, $1 \mathrm{H}), 12.5$ (s, $1 \mathrm{H}, \mathrm{N}-\mathrm{H})$.

\section{2-(4-Chlorophenyl)-4, 5-diphenyl-1H-imidazole (Table 2, entry 6)}

FT-IR: $\bar{v}(\mathrm{KBr})=3026,1600,1482,1069,826,766,695 \mathrm{~cm}^{-1},{ }^{1} \mathrm{H}-\mathrm{NMR}\left(400 \mathrm{MHz}, \mathrm{DMSO}-d_{6}\right.$, ppm): 12.8(s, $1 \mathrm{H}, \mathrm{N}-\mathrm{H}), 8.14(\mathrm{~s}, 1 \mathrm{H}, \mathrm{C}-\mathrm{H}), 8.04$ (d, $\left.1 \mathrm{H},{ }^{3} \mathrm{~J}=7 \mathrm{~Hz}\right), 7.25-7.55$ (m, $\left.12 \mathrm{H}, \mathrm{Ar}-\mathrm{H}\right)$.

\section{2-(4-Bromophenyl)-4, 5-diphenyl-1H-imidazole (Table 2, entry 8)}

FT-IR: $\bar{v}(\mathrm{KBr})=2963,1452,1490,835,764,694 \mathrm{~cm}^{-1},{ }^{1} \mathrm{H}-\mathrm{NMR}\left(400 \mathrm{MHz}, \mathrm{DMSO}-d_{6}, \mathrm{ppm}\right): 7.35$ (brs, $7 \mathrm{H}$ ), 7.56 (brs, $3 \mathrm{H}$ ), 7.61(brs, $4 \mathrm{H}$ ). 


\section{2-(4-Nitrophenyl)-4, 5-diphenyl-1H-imidazole (Table 2, entry 11)}

FT-IR: $\bar{v}(\mathrm{KBr})=3061,1599,1487,1516,1339,1108,854,765,695 \mathrm{~cm}^{-1},{ }^{1} \mathrm{H}-\mathrm{NMR}(400 \mathrm{MHz}$, DMSO- $d_{6}$, ppm): 7.29 (brs, $\left.3 \mathrm{H}\right), 7.40$ (brs, $3 \mathrm{H}$ ), 7.52 (brs, $\left.2 \mathrm{H}\right), 7.67$ (d, $\left.{ }^{3} J=8,4 \mathrm{H}\right), 8.07$ (m, $\left.2 \mathrm{H}\right)$, 11.81(s, $1 \mathrm{H}, \mathrm{N}-\mathrm{H})$.

\section{4, 5-Diphenyl-1H-imidazole (Table 2, entry 12)}

Yield: $43 \%$, white solid, m.p. $101-103{ }^{\circ} \mathrm{C}$.

FT-IR: $\bar{v}(\mathrm{KBr})=3436,3050,1600,1460,1127,765,697 \mathrm{~cm}-1,{ }^{1} \mathrm{H}-\mathrm{NMR}$ (500 MHz, DMSO-d6): 12.68 (s, 1H, N-H), 8.08 (d, 3J = 6 Hz, 2 H), 7.46-7.54 (m, 4 H, Ar-C-H), 7.38 (brs, 2 H, Ar-C-H), 7.30 (brs, 2 H, Ar-C-H), 7.22 (brs, $1 \mathrm{H}$ ) ppm. Elemental analysis. Found, \%: C 81.68; H 5.55; N 12.77. $\mathrm{C}_{15} \mathrm{H}_{12} \mathrm{~N}_{2}$. Calculated, \%: C 81.79; H 5.49; N 12.72 .

\section{References}

1. Eyers, P. A.; Craxton, M.; Morrice, N.; Cohen, P.; Goedert. M. (1998) Conversion of SB 203580-insensitive MAP kinase family members to drug-sensitive forms by a single aminoacid substitution. Chem. Biol., 5, 321-328.

2. Antolini, M.; Bozzoli, A.; Ghiron, C.; Kennedy, G.; Rossi, T.; Ursini, A. (1999) Analogues of 4,5-bis(3,5-dichlorophenyl)-2-trifluoromethyl-1H-imidazole as potential antibacterial agents. Bioorg. Med. Chem. Lett., 9, 1023-1028.

3. Wang, L.; Woods, K. W.; Li, Q.; Barr, K. J.; Mc Croskey, R. W.; Hannick, S. M.; Gherke L, Credo RB, Hui Y-H, Marsh K, Warner R, Lee JY, Zielinsky-Mozng, N.; Frost, D.; Rosenberg, S. H.; Sham, H. L. (2002) Potent, orally active heterocycle-based combretastatin A-4 analogues: synthesis, structure-activity relationship, pharmacokinetics, and in vivo antitumor activity evaluation J. Med. Chem., 45, 1697-1711.

4. De Laszlo, S. E.; Hacker, C.; Li, B.; Kim, D.; Mac Coss, M.; Mantl, N.; Pivnichny, J. V.; Colwell, L.; Koch, G. E.; Cascieri, M. A.; Hagmann, W. K. (1999) Potent, orally absorbed glucagon receptor antagonists, Bioorg. Med. Chem. Lett. 9, 641-646.

5. Lambardino, J. C.; Wiseman, E. H. (1974) Preparation and anti inflammatory activity of some nonacidic tri-substituted imidazoles. J. Med. Chem. 17, 1182-1188.

6. Lantos, I.; Zhang, W. Y.; Shiu, X.; Eggleston, D. S. (1993) Synthesis of imidazoles via heteroCope rearrangements J. Org. Chem. 58, 7092-7095.

7. Sharma, G.V.; Jyothi, Y.; Lakshmi, P.S. (2006) Efficient room-temperature synthesis of tri- and tetrasubstituted imidazoles catalyzed by $\mathrm{ZrCl}_{4}$, Synth. Commun., 36, 2991-3000.

8. Siddiqui, S. A.; Narkhede, U. C.; Palimkar, S. S.; Daniel, T.; Lahoti, R. J.; Srinivasan, K. V. (2005) Room temperature ionic liquid promoted improved and rapid synthesis of 2,4,5-tri-aryl imidazoles from aryl aldehydes and 1, 2-diketones or $\alpha$-hydroxyketone. Tetrahedron 61, 35393546.

9. Balalaei, S.; Arabanian, A. (2000) One-pot synthesis of tetrasubstituted imidazoles catalyzed by zeolite HY and silica gel under microwave irradiation, Green Chem. 2, 274-276.

10. Sharma, S.D.; Hazarika, P.; Konwar, D. (2008) An efficient and one-pot synthesis of 2,4,5trisubstituted and 1, 2,4,5-tetrasubstituted imidazoles catalyzed by $\mathrm{InCl}_{3} \cdot 3 \mathrm{H}_{2} \mathrm{O}$, Tetrahedron Lett. 49, 2216-2220.

11. Murthy, S.N.; Madhav, B.; Nageswar, Y.V.D. (2010) DABCO as a mild and efficient catalytic system for the synthesis of highly substituted imidazoles via multi-component condensation strategy, Tetrahedron Lett. 51, 5252-5257.

12. Wang, X. C.; Gong, H.P.; Quan, Z.J.; Li, L.; Ye, H.L. (2009) PEG-400 as an efficient reaction medium for the synthesis of 2,4,5-triaryl- $1 \mathrm{H}$-imidazoles and 1, 2,4,5-tetra-aryl-1H-imidazoles. Chinese Chem. Lett. 20, 44-47. 
13. Karimi, A. R.; Alimohamadi, Z.; Amini, M. M. (2010) Wells-Dawson heteropolyacid supported on silica: a highly efficient catalyst for synthesis of 2,4,5-trisubstituted and 1,2,4,5tetrasubstituted imidazoles, Mol. Divers. 14, 635-641.

14. Zang, H.; Su, Q.; Mo, Y.; Cheng, B.-W. Jun, S. (2010) Ionic liquid [EMIM]OAc under ultrasonic irradiation towards the first synthesis of trisubstituted imidazoles. Ultrason. Sonochem., 17, 749-751.

15. Karimi-Jaberi, Z.; Barekat, M. (2010) One-pot synthesis of tri-and tetra-substituted imidazoles using sodium dihydrogen phosphate under solvent-free conditions.Chinese Chem. Lett. 21, $1183-1186$.

16. Shaterian, H. R.; Ranjbar, M. (2011) An environmental friendly approach for the synthesis of highly substituted imidazoles using Brønsted acidic ionic liquid, $N$-methyl-2-pyrrolidonium hydrogen sulfate, as reusable catalyst. J. Mol. Liquid. 160, 40-49.

17. Teimouri, A.; Najafi Chermahini, A. (2011) An efficient and one-pot synthesis of 2,4,5trisubstituted and 1, 2,4,5-tetrasubstituted imidazoles catalyzed via solid acid nano-catalyst. $J$. Mol. Catal. A: Chem. 346, 39-45.

18. Chary, M.V.; Keerthysri, N.C.; Vupallapati, S.V.N.; Lingaiah,N.; Kantevari, S. (2008) Tetrabutylammonium bromide (TBAB) in isopropanol: An efficient, novel, neutral and recyclable catalytic system for the synthesis of 2,4,5-trisubstituted imidazoles. Catal. Commun. 9, 2013-2017.

19. Safari, J.; DehghanKhalili, S.; Banitaba, S. H. (2010) A novel and an efficient catalyst for onepot synthesis of 2,4,5-trisubstituted imidazoles by using microwave irradiation under solventfree conditions. J. Chem. Sci., 122, 437-441.

20. Safari, J.; DehghanKhalili, S.; Rezaei, M.; Banitaba, S. H. Meshkani, F. (2010) Nanocrystalline Magnesium Oxide: A novel and efficient catalyst for facile synthesis of 2,4,5trisubstituted imidazole derivatives (III). Monatsh. Chem. 141, 1339-1345.

21. Joshi, R.S.; Mandhane, P. G.; Shaikh,M. U.; Kale, R. P.; Gill, C. H. (2010) Potassium dihydrogen phosphate catalyzed one-pot synthesis of 2,4,5-triaryl-1H-imidazoles. Chinese Chem. Lett., 21, 429-431

22. Bamoniri, A.; Zolfigol, M. A.; Mirjalili, B. F.; Fallah, F. (2007) Efficient procedure for chemoselective n-nitrosation of secondary amines with trichloromelamine- $\mathrm{NaNO}_{2}$ Russian $\mathrm{J}$. Org. Chem., 43, 1393-1396

23. Mirjalili, B. F.; Bamoniri, A.; Akrami, H. R. (2009) Trichloromelamine/ $\mathrm{Zr}\left(\mathrm{HSO}_{4}\right)_{4} / \mathrm{KBr}$ : an efficient heterogeneous alternative for oxidation of alcohols and diols. Digest J. Nanomat. Biostruct., 4, 763-767.

24. Mirjalili, B. F.; Bamoniri, A.; Amrollahi, M. A.; Dastan, E. (2009) Trichloromelamine and triphenylphosphine as a versatile and efficient system for oxidation of alcohols under solventfree condition. Iran. J. Org. Chem. 1, 13-17.

25. Ghorbani-Ch, A.; Amani, K.; Zolfigol, M. A.; Hajjami, M.; Ayazi-Nasrabadi, R. (2009) 1, 3Dichloro-5, 5-dimethylhydantoin (DCH) and trichloromelamine (TCM) as efficient catalysts for the chemoselective trimethylsilylation of hydroxyl group with 1, 1,1,3,3,3hexamethyldisilazane (HMDS) under mild conditions. J. Chinese Chem. Soc., 56, 255-260.

26. Mirjalili, B.F.; Bamoniri, A.; Akbari. (2008) $\mathrm{BF}_{3} \cdot \mathrm{SiO}_{2}$ : an efficient alternative for the synthesis of 14-aryl or alkyl-14H-dibenzo[a,j]xanthenes. Tetrahedron Lett., 49, 6454-6456.

27. Sadeghi, B.; Mirjalili, B. F.; Hashemi, M.M. (2008) $\mathrm{BF}_{3} \cdot \mathrm{SiO}_{2}$ : An efficient heterogeneous alternative for regio-chemo and stereoselective claisen-schmidt condensation. J. Iran. Chem. Soc., 5, 694-698.

28. Mirjalili, B.F.; Bamoniri, A.; Akbari, A. (2011) One-Pot synthesis of 3, 4-dihydropyrimidin2(1H)-ones (thiones) promoted by nano-BF $\mathrm{B}_{3} \cdot \mathrm{SiO}_{2}$. J. Iran. Chem. Soc., 8, S135-S140.

29. Sadeghi, B.; Mirjalili, B.F.; Hashemi, M.M. (2008) $\mathrm{BF}_{3} \cdot \mathrm{SiO}_{2}$ : an efficient reagent system for the one-pot synthesis of 1, 2,4,5-tetrasubstituted imidazoles. Tetrahedron Lett., 49, 2575-2577. 


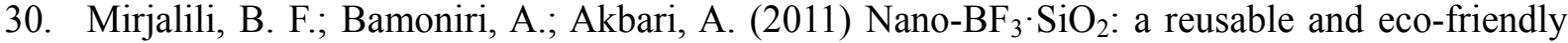
catalyst for synthesis of quinoxalines. Chem. Heterocycl. Comp., 47, 487-491.

31. Samai, S.; Nandi, G.C.;Singh, P.; Singh, M.S. (2009) L-Proline: an efficient catalyst for the one-pot synthesis of 2,4,5-trisubstituted and 1, 2,4,5-tetrasubstituted imidazoles. Tetrahedron, $65,10155-10161$. 